\title{
EXPECTED RIESZ ENERGY OF SOME DETERMINANTAL PROCESSES ON FLAT TORI
}

\author{
JORDI MARZO AND JOAQUIM ORTEGA-CERDÀ
}

\begin{abstract}
We compute the expected Riesz energy of random points on flat tori drawn from certain translation invariant determinantal processes and determine the process in the family providing the optimal asymptotic expected Riesz energy.
\end{abstract}

\section{INTRODUCTION}

Our objective is to study the asymptotics of the expected Riesz energy of certain point processes (random finite point configurations) in a flat torus $\Omega \subset \mathbb{R}^{d}$. If $\Lambda$ is a lattice in $\mathbb{R}^{d}$ (i.e. $\Lambda=A \mathbb{Z}^{d}$ for some nonsingular square matrix $A$ ) we identify the fundamental domain

$$
\Omega=\left\{t_{1} v_{1}+\cdots+t_{d} v_{d}: t_{1}, \ldots, t_{d} \in[0,1)\right\},
$$

where the column vectors of $A=\left[v_{1}, \ldots, v_{d}\right]$ is a $\mathbb{Z}$ basis of the lattice $\Lambda$, with the flat torus $\mathbb{R}^{d} / \Lambda$.

For the sphere $\mathbb{S}^{2}$, the authors in [1] estimate asymptotically the expected energy of points of the, so-called, spherical ensemble. In [3], the authors study the harmonic ensemble in $\mathbb{S}^{d}$ and prove, in some cases, the optimality of the expected asymptotic energy of this process among rotation invariant determinantal processes. In both cases, the expected asymptotic energy was used to get upper bounds for the minimal Riesz energy. Here, we study also the optimality of the expected asymptotic energy among a collection of determinantal processes invariant under translations and it turns out that the best process can be found as an easy consequence of Riesz's rearrangement inequality.

This provides explicit examples with the lowest energy bounds known on the torus in high dimensions.

1.1. Riesz energy. To define the Riesz energy in this periodic setting we follow [6, 7], see also [5, Section 9]. Given a lattice $\Lambda=A \mathbb{Z}^{d} \subset \mathbb{R}^{d}$ the Epstein Hurwitz

Key words and phrases. Riesz energy, Epstein zeta function, Determinantal processes, Torus, Rearrangement inequality.

This research has been partially supported by the MTM2014-51834-P grant by the Ministerio de Economía y Competitividad, Gobierno de España and by the Generalitat de Catalunya (project 2014 SGR 289). 
zeta function for $\Lambda$ is defined, for $s>d$, as

$$
\zeta_{\Lambda}(s ; x)=\sum_{v \in \Lambda} \frac{1}{|x+v|^{s}}, \quad x \in \mathbb{R}^{d} .
$$

Observe that $\zeta_{\Lambda}(s ; x)$ is the $\Lambda$-periodic potential generated by the Riesz s-energy $|x|^{-s}$.

When $s \leq d$ the sum above is infinite for all $x \in \mathbb{R}^{d}$. For fixed $x \in \mathbb{R}^{d} \backslash \Lambda$ define the function

$F_{s, \Lambda}(x)=\sum_{v \in \Lambda} \int_{1}^{+\infty} e^{-|x+v|^{2} t} \frac{t^{\frac{s}{2}-1}}{\Gamma\left(\frac{s}{2}\right)} d t+\frac{1}{|\Lambda|} \sum_{w \in \Lambda^{*} \backslash\{0\}} e^{2 \pi i\langle x, w\rangle} \int_{0}^{1} \frac{\pi^{d / 2}}{t^{d / 2}} e^{-\frac{\pi^{2}|w|^{2}}{t}} \frac{t^{\frac{s}{2}-1}}{\Gamma\left(\frac{s}{2}\right)} d t$

where $\Lambda^{*}=\left\{x \in \mathbb{R}^{d}: \forall \lambda \in \Lambda \quad\langle x, \lambda\rangle \in \mathbb{Z}\right\}=\left(A^{t}\right)^{-1} \mathbb{Z}^{d}$ is the dual lattice and $|\Lambda|=|\operatorname{det} A|$ is the co-volume of $\Lambda$.

Then, $F_{s, \Lambda}(x)$ is an entire function of $s$ and therefore by the relation

$$
F_{s, \Lambda}(x)=\zeta_{\Lambda}(s ; x)+\frac{2 \pi^{d / 2}|\Lambda|^{-1}}{\Gamma\left(\frac{s}{2}\right)(d-s)}, \quad s>d,
$$

we obtain a analytic continuation of $\zeta_{\Lambda}(s ; x)$ to $s \in \mathbb{C} \backslash\{d\}$. Observe that the function $1 / \Gamma(s)$ is entire and that all the sums in (1) converge uniformly. We are interested in the range $0<s<d$.

For $\omega=\left(x_{1}, \ldots, x_{N}\right) \in \Omega^{N}$ define, for $0<s<d$, the periodic Riesz $s$-energy of $\omega$ by

$$
E_{s, \Lambda}(\omega)=\sum_{k \neq j} F_{s, \Lambda}\left(x_{k}-x_{j}\right)
$$

and the minimal periodic Riesz $s$-energy by

$$
\mathcal{E}_{s, \Lambda}(N)=\inf _{\omega \in\left(\mathbb{R}^{d}\right)^{N}} E_{s, \Lambda}(\omega) .
$$

1.2. Determinantal processes. For the introductory background we follow 8 , Chap. 4].

We denote as $\mathcal{X}$ a (simple) random point process in a compact set $\Omega \subset \mathbb{R}^{d}$. And let $\mu$ be the normalized Lebesgue measure. A way to describe the process is to specify the random variable counting the number of points of the process in $D$, for all Borel sets $D \subset \Omega$. We denote this random variable as $\mathcal{X}(D)$.

These point processes are characterized by their joint intensity functions $\rho_{k}$ in $\Omega^{k}$ satisfying that

$$
\mathbb{E}\left[\mathcal{X}\left(D_{1}\right) \cdots \mathcal{X}\left(D_{k}\right)\right]=\int_{D_{1} \times \cdots \times D_{k}} \rho_{k}\left(x_{1}, \ldots, x_{k}\right) d \mu\left(x_{1}\right) \ldots d \mu\left(x_{k}\right),
$$

for any family of mutually disjoint subsets $D_{1}, \ldots, D_{k} \subset \Omega$. We assume that $\rho_{k}\left(x_{1}, \ldots, x_{k}\right)=0$ when $x_{i}=x_{j}$ for $i \neq j$. 
A random point process is called determinantal with kernel $K: \Omega \times \Omega \rightarrow \mathbb{C}$, if it is simple and the joint intensities with respect to a background measure $\mu$ are given by

$$
\rho_{k}\left(x_{1}, \ldots, x_{k}\right)=\operatorname{det}\left(K\left(x_{i}, x_{j}\right)\right)_{1 \leq i, j \leq k},
$$

for every $k \geq 1$ and $x_{1}, \ldots, x_{k} \in \Omega$.

To define the processes we will consider only projection kernels.

Definition 1. We say that $K$ is a projection kernel if it is a Hermitian projection kernel, i.e. the integral operator in $L^{2}(\mu)$ with kernel $K$ is self-adjoint and has eigenvalues 1 and 0 .

By Macchi-Soshnikov's theorem [8, Theorem 4.5.5], a projection kernel $K(x, y)$ defines a determinantal process and it has $N$ points almost surely if the trace for the corresponding integral operator equals $N$, i.e. if

$$
\int_{\Omega} K(x, x) d \mu(x)=N
$$

Observe that the random vector in $\Omega^{N}$ generated with density

$$
\frac{1}{N !} \operatorname{det}\left(K\left(x_{i}, x_{j}\right)\right)_{1 \leq i, j \leq k},
$$

is a determinantal process with the right marginals i.e. the joint intensities are given by determinants of the kernel [2, Remark 4.2.6].

Given now a function $f: \Omega \times \Omega \rightarrow[0, \infty)$ it is easy to compute the expected pair potential energy, [8, Formula (1.2.2)]:

Proposition 1. Let $K(x, y)$ be a projection kernel with trace $N$ in $\Omega$ and let $\omega=\left(x_{1}, \ldots, x_{N}\right) \in \Omega^{N}$ be $N$ random points generated by the corresponding determinantal point process. Then, for any measurable $f: \Omega \times \Omega \rightarrow[0, \infty)$ we have

$\mathbb{E}_{\omega \in \Omega^{N}}\left(\sum_{i \neq j} f\left(x_{i}, x_{j}\right)\right)=\int_{x, y \in \Omega}\left(K(x, x) K(y, y)-|K(x, y)|^{2}\right) f(x, y) d \mu(x) d \mu(y)$.

1.2.1. Flat torus. In our setting we take as $\Omega \subset \mathbb{R}^{d}$ the flat torus $\mathbb{R}^{d} / \Lambda$, for some lattice $\Lambda$ with dual $\Lambda^{*}$.

To construct the kernel we consider for $w \in \Lambda^{*}$, the Laplace-Beltrami eigenfunctions $f_{w}(u)=e^{2 \pi i\langle u, w\rangle}$ of eigenvalue $-4 \pi^{2}\langle w, w\rangle$. Then

$$
\Delta f_{w}+4 \pi^{2}\langle w, w\rangle f_{w}=0,
$$

and $\left\{f_{w}\right\}_{w \in \Lambda^{*}}$ are orthonormal in $L^{2}(\Omega)$, with respect to the normalized Lebesgue measure $\mu$ in $\Omega$

$$
\int_{\Omega} f_{w}(u) \overline{f_{w^{\prime}}(u)} d \mu(u)=\delta_{w, w^{\prime}}
$$

for $w, w^{\prime} \in \Lambda^{*}$. 
Now, we consider functions $\kappa=\left(\kappa_{N}\right)_{N \geq 0}$ where each $\kappa_{N}: \Lambda^{*} \longrightarrow\{0,1\}$ has compact support and we define the kernels

$$
K_{N}(u, v)=\sum_{w \in \Lambda^{*}} \kappa_{N}(w) e^{2 \pi i\langle u-v, w\rangle}, \quad u, v \in \Omega,
$$

and the corresponding determinantal point processes on the flat torus $\Omega$. For these processes (we are not going to distinguish between the integral operator defined by the kernel $K_{N}$ and the kernel itself) we get

$$
\operatorname{tr}\left(K_{N}\right)=\int_{\Omega} K_{N}(u, u) d \mu(u)=\sum_{w \in \Lambda^{*}} \kappa_{N}(w)=\# \operatorname{supp} \kappa_{N},
$$

points almost surely.

1.2.2. Examples. Let $\Lambda=\mathbb{Z}^{d}$. The flat torus $\mathbb{T}^{d}=\mathbb{R}^{d} / \mathbb{Z}^{d}$ has $\Omega=[0,1)^{d}$.

Consider

$$
\kappa_{N}(x)=\chi_{[-N, N]^{d}}(x), \quad x \in \mathbb{R}^{d}
$$

then for $u, v \in[0,1)^{d}$

$$
K_{N}(u, v)=\sum_{\lambda \in \mathbb{Z}^{d},\|\lambda\|_{\infty} \leq N} e^{2 \pi i\langle u-v, \lambda\rangle}=\prod_{j=1}^{d} D_{N}\left(u_{j}-v_{j}\right),
$$

where

$$
D_{N}(x)=\frac{\sin \left[\left(N+\frac{1}{2}\right) x\right]}{\sin \left(\frac{x}{2}\right)}, x \in \mathbb{T},
$$

is the Dirichlet kernel.

If $N \in \mathbb{N}$ can be expressed as a sum $N=\lambda_{1}^{2}+\cdots+\lambda_{d}^{2}$ for $\lambda \in \mathbb{Z}^{d}$. We can define

$$
K_{N}(u, v)=\sum_{\lambda \in \mathbb{Z}^{d},\|\lambda\|_{2}^{2}=N} e^{2 \pi i\langle u-v, \lambda\rangle}, \quad u, v \in[0,1)^{d} .
$$

Observe that $\left\{e^{2 \pi i\langle\cdot, \lambda\rangle}\right\}_{\lambda \in \mathbb{Z}^{d},\|\lambda\|_{2}^{2}=N}$ span the eigenspace corresponding to eigenvalue $-4 \pi^{2} N$ and it has dimension $r_{d}(N)$. Where $r_{d}(N)$ is the number of different ways that $N$ may be expressed as a sum of $d$ squares (the order in the sum of the squares is counted as distinct). For example $r_{2}(4)=4$ because $4=0+2^{2}=0+(-2)^{2}=2^{2}+0=(-2)^{2}+0$. This corresponds to the four distinct points $(0, \pm 2)$ and $( \pm 2,0)$.

1.3. Some known results about minimal periodic Riesz $s$-energy. It was shown in [7] that for $0<s<d$ there exists a constant $C_{s, d}$ independent of $\Lambda$ such that for $N \rightarrow \infty$

$$
\mathcal{E}_{s, \Lambda}(N)=\frac{2 \pi^{d / 2}|\Lambda|^{-1}}{\Gamma\left(\frac{s}{2}\right)(d-s)} N^{2}+C_{s, d}|\Lambda|^{-s / d} N^{1+\frac{s}{d}}+o\left(N^{1+\frac{s}{d}}\right) .
$$


The constant $C_{s, d}$ above is not known (unless $d=1$ ). In [7] the authors found an upper bound in terms of the Epstein zeta function. Recall that for a lattice $\Lambda \subset \mathbb{R}^{d}$, the Epstein zeta function $\zeta_{\Lambda}(s)$ defined by

$$
\zeta_{\Lambda}(s)=\sum_{v \in \Lambda \backslash\{0\}} \frac{1}{|v|^{s}}, \quad s>d,
$$

can be extended analytically (as in (1D) to $\mathbb{C} \backslash\{d\}$. One can see easily that $\zeta_{\Lambda}(0)=-1$ and the residue of $\zeta_{\Lambda}(s)$ in $d$ is $2 \pi^{d / 2} / \Gamma(d / 2)=\omega_{d-1}$. The result in [7, Corollary 3] is that for $0<s<d$

$$
C_{s, d} \leq \inf _{\Lambda} \zeta_{\Lambda}(s)
$$

where $\Lambda$ runs on the lattices with $|\Lambda|=1$. It has been conjectured (see [4]) that if $d=2,4,8$, or 24 , then $C_{s, d}=\zeta_{\Lambda_{d}}(s)$ where $\Lambda_{d}$ denotes (respectively) the hexagonal lattice, the $D_{4}$ lattice, the $E_{8}$ lattice and the Leech lattice (scaled to have $\left.\left|\Lambda_{d}\right|=1\right)$. When $d=1$ indeed $C_{s, 1}=\zeta_{\mathbb{Z}}(s)=2 \zeta(s)$. For $d=2$ it is known, due to the work of several authors, that $\inf _{\Lambda} \zeta_{\Lambda}(s)$ is attained for the triangular lattice, see [11] where the result is deduced from the corresponding result for theta functions. It is observed in [12] that from Siegel's integration formula it follows that

$$
\int \zeta_{\Lambda}(s) d \lambda_{d}(\Lambda)=0
$$

where $d \lambda_{d}$ is the volume measure in the space of lattices, [14, p. 172]. One deduces then that $C_{s, d}<0$, although for large dimensions there are no examples providing negative bounds. Indeed, from [12], see also [13, Theorem 1], all explicitly known lattices in large dimensions are such that the corresponding Epstein zeta functions have a zero in $0<s<d$, i.e. the analogue of the Riemann hypothesis fails for Epstein zeta functions, see Remark 2.

\section{EXPECTED ENERGIES}

By Proposition 1 the expected periodic Riesz s-energy of $t_{N}=\operatorname{tr}\left(K_{N}\right)$ random points $\omega=\left(x_{1}, \ldots, x_{t_{N}}\right)$ drawn from the determinantal process defined by the kernel $K_{N}(u, v)$ is

$\mathbb{E}_{\omega \in\left(\mathbb{R}^{d}\right)^{t_{N}}}\left(E_{s, \Lambda}(\omega)\right)=\int_{\Omega^{2}}\left(K_{N}(u, u) K_{N}(v, v)-\left|K_{N}(u, v)\right|^{2}\right) F_{s, \Lambda}(u-v) d \mu(u) d \mu(v)$.

It is easy to see, [6], that for $0<s<d$

$$
\int_{\Omega^{2}} F_{s, \Lambda}(u-v) d \mu(u) d \mu(v)=\frac{2 \pi^{d / 2}|\Lambda|^{-1}}{\Gamma\left(\frac{s}{2}\right)(d-s)},
$$

and therefore by translation invariance

$$
\mathbb{E}_{\omega \in\left(\mathbb{R}^{d}\right)^{t} N}\left(E_{s, \Lambda}(\omega)\right)=\frac{2 \pi^{d / 2}|\Lambda|^{-1}}{\Gamma\left(\frac{s}{2}\right)(d-s)} t_{N}^{2}-\int_{\Omega}\left|K_{N}(u, 0)\right|^{2} F_{s, \Lambda}(u) d \mu(u) .
$$


Our first result is a nice closed expression for the integral above.

Theorem 1. Let $\omega=\left(x_{1}, \ldots, x_{t_{N}}\right)$ be drawn from the determinantal process on the flat torus $\mathbb{R}^{d} / \Lambda$ given by the kernel

$$
K_{N}(u, v)=\sum_{w \in \Lambda^{*}} \kappa_{N}(w) e^{2 \pi i\langle u-v, w\rangle},
$$

with $\kappa_{N}(w) \in\{0,1\}$ for $w \in \Lambda^{*}$ and $\sum_{w \in \Lambda^{*}} \kappa_{N}(w)=t_{N}$.

Then, for $0<s<d$,

$\mathbb{E}_{\omega \in\left(\mathbb{R}^{d}\right)^{t_{N}}}\left(E_{s, \Lambda}(\omega)\right)=\frac{2 \pi^{d / 2}}{\Gamma\left(\frac{s}{2}\right)(d-s)|\Lambda|}\left(t_{N}{ }^{2}-t_{N}\right)-\frac{\pi^{s-\frac{d}{2}} \Gamma\left(\frac{d-s}{2}\right)}{\Gamma\left(\frac{s}{2}\right)|\Lambda|} \sum_{\substack{w, w^{\prime} \in \Lambda^{*} \\ w \neq w^{\prime}}} \frac{\kappa_{N}(w) \kappa_{N}\left(w^{\prime}\right)}{\left|w-w^{\prime}\right|^{d-s}}$.

Remark 1. Observe that for $N$ random points chosen independently and uniformly in $\Omega$ (i.e. for the Poisson point process) the expected energy is given by

$$
\mathbb{E}_{\text {uniform }}\left(E_{s, \Lambda}\right)=\frac{2 \pi^{d / 2}|\Lambda|^{-1}}{\Gamma\left(\frac{s}{2}\right)(d-s)}\left(N^{2}-N\right)
$$

so the improvement (lowering) in the determinantal case comes from the last summand above. Therefore, to get a good upper bound for the minimal energy we want to maximize the sum

$$
\sum_{\substack{w, w^{\prime} \in \Lambda^{*} \\ w \neq w^{\prime}}} \frac{\kappa_{N}(w) \kappa_{N}\left(w^{\prime}\right)}{\left|w-w^{\prime}\right|^{d-s}}, \text { given } \sum_{w \in \Lambda^{*}} \kappa_{N}(w)=t_{N}
$$

This is not an easy task in general. For example, when $t_{N}=2$ this would lead to find the shortest non-zero vector in the lattice $\Lambda^{*}$ i.e.

$$
m\left(\Lambda^{*}\right)=\min \left\{|w|: w \in \Lambda^{*} \backslash\{0\}\right\},
$$

or equivalently, the density of the densest lattice sphere packing.

Proof. To compute the integral in (3) we write

$$
\left|K_{N}(u, 0)\right|^{2}=\sum_{w, w^{\prime} \in \Lambda^{*}} \kappa_{N}(w) \kappa_{N}\left(w^{\prime}\right) e^{2 \pi i\left\langle u, w-w^{\prime}\right\rangle},
$$

and using the expression for $F_{s, \Lambda}(u)$, where the sums converge uniformly, we get

$$
\sum_{v \in \Lambda} \int_{\Omega} e^{-|u+v|^{2} t} e^{2 \pi i\left\langle u, w-w^{\prime}\right\rangle} d u=\int_{\mathbb{R}^{d}} e^{-|u|^{2} t} e^{2 \pi i\left\langle u, w-w^{\prime}\right\rangle} d u=\left(\frac{\pi}{t}\right)^{d / 2} e^{-\pi^{2} \frac{\left|w-w^{\prime}\right|^{2}}{t}}
$$

and

$\frac{1}{|\Lambda|} \sum_{w, w^{\prime} \in \Lambda^{*}} \kappa_{N}(w) \kappa_{N}\left(w^{\prime}\right) \sum_{\eta \in \Lambda^{*} \backslash\{0\}} \int_{0}^{1}\left(\frac{\pi}{t}\right)^{d / 2} e^{-\pi^{2} \frac{|\eta|^{2}}{t}} \frac{t^{\frac{s}{2}-1}}{\Gamma\left(\frac{s}{2}\right)}\left[\int_{\Omega} e^{2 \pi i\left\langle u, w-w^{\prime}+\eta\right\rangle} d \mu(u)\right] d t$.

Observe that

$$
\int_{\Omega} e^{2 \pi i\left\langle u, w-w^{\prime}+\eta\right\rangle} d \mu(u)=\delta_{w^{\prime}-w, \eta}
$$


SO

$$
\frac{1}{|\Lambda|} \sum_{\substack{w, w^{\prime} \in \Lambda^{*} \\ w \neq w^{\prime}}} \kappa_{N}(w) \kappa_{N}\left(w^{\prime}\right) \int_{0}^{1}\left(\frac{\pi}{t}\right)^{d / 2} e^{-\pi^{2} \frac{\left|w-w^{\prime}\right|^{2}}{t}} \frac{t^{\frac{s}{2}-1}}{\Gamma\left(\frac{s}{2}\right)} d t .
$$

Putting all together, and using that for $s<d$

$$
\int_{1}^{+\infty}\left(\frac{\pi}{t}\right)^{d / 2} t^{\frac{s}{2}-1} d t=\frac{2 \pi^{d / 2}}{d-s}
$$

we get

$$
\begin{gathered}
\int_{\Omega}\left|K_{N}(u, 0)\right|^{2} F_{s, \Lambda}(u) d \mu(u)=\frac{1}{|\Lambda|} \sum_{w, w^{\prime} \in \Lambda^{*}} \kappa_{N}(w) \kappa_{N}\left(w^{\prime}\right) \int_{1}^{+\infty}\left(\frac{\pi}{t}\right)^{d / 2} e^{-\pi^{2} \frac{\left|w-w^{\prime}\right|^{2}}{t}} \frac{t^{\frac{s}{2}}-1}{\Gamma\left(\frac{s}{2}\right)} d t \\
+\frac{1}{|\Lambda|} \sum_{\substack{w, w^{\prime} \in \Lambda^{*} \\
w \neq w^{\prime}}} \kappa_{N}(w) \kappa_{N}\left(w^{\prime}\right) \int_{0}^{1}\left(\frac{\pi}{t}\right)^{d / 2} e^{-\pi^{2} \frac{\left|w-w^{\prime}\right|^{2}}{t}} \frac{t^{\frac{s}{2}-1}}{\Gamma\left(\frac{s}{2}\right)} d t \\
=\frac{2 \pi^{d / 2} t_{N}}{(d-s) \Gamma\left(\frac{s}{2}\right)|\Lambda|}+\frac{1}{|\Lambda|} \sum_{\substack{w, w^{\prime} \in \Lambda^{*} \\
w \neq w^{\prime}}} \kappa_{N}(w) \kappa_{N}\left(w^{\prime}\right)\left[\int_{0}^{+\infty}\left(\frac{\pi}{t}\right)^{d / 2} e^{-\pi^{2} \frac{\left|w-w^{\prime}\right|^{2}}{t}} \frac{t^{\frac{s}{2}-1}}{\Gamma\left(\frac{s}{2}\right)} d t\right]
\end{gathered}
$$

This last integral converges for all $s<d$, and using that (for $\alpha<-1$ )

$$
\int_{0}^{+\infty} e^{-c^{2} / t} t^{\alpha} d t=c^{2 \alpha+2} \Gamma(-\alpha-1)
$$

we get the result.

Now we define a way to get different invariant kernels (by choosing different sequences of functions $\kappa$ ) and we estimate the corresponding expected energies.

Definition 2. Let $\mathcal{D} \subset \mathbb{R}^{d}$ be an open bounded subset with boundary of measure zero and let $\Lambda \subset \mathbb{R}^{d}$ be a lattice. Define for $N \in \mathbb{N}$ the functions $\kappa^{\mathcal{D}, \Lambda}=\left(\kappa_{N}\right)_{N \geq 0}$ where

$$
\kappa_{N}(w)= \begin{cases}1 & \text { if } w \in \Lambda^{*} \cap N^{1 / d} \mathcal{D} \\ 0 & \text { otherwise. }\end{cases}
$$

Proposition 2. Let $\Lambda \subset \mathbb{R}^{d}$ be a lattice and $\mathcal{D} \subset \mathbb{R}^{d}$ be an open bounded subset with boundary of measure zero and such that $|\Lambda||\mathcal{D}|=1$. Let $\kappa^{\mathcal{D}, \Lambda}=\left(\kappa_{N}\right)_{N \geq 0}$ be defined as above. Suppose that the trace of the corresponding kernel equals $t_{N} i . e$.

$$
\sum_{w \in \Lambda^{*}} \kappa_{N}(w)=t_{N}
$$

Then, for $0<s<d$, if $\omega=\left(x_{1}, \ldots, x_{t_{N}}\right) \in \Omega^{t_{N}}$ are $t_{N}$ points drawn from the determinantal process defined by $\kappa^{\mathcal{D}, \Lambda}$

$$
\mathbb{E}_{\omega \in\left(\mathbb{R}^{d}\right)^{t} N}\left(E_{s, \Lambda}(\omega)\right)=\frac{2 \pi^{d / 2}|\Lambda|^{-1}}{\Gamma\left(\frac{s}{2}\right)(d-s)} t_{N}^{2}-\frac{\pi^{s-\frac{d}{2}} \Gamma\left(\frac{d-s}{2}\right)}{\Gamma\left(\frac{s}{2}\right)|\Lambda|} I_{\nu}^{\mathcal{D}}(d-s) t_{N}^{1+s / d}+o\left(t_{N}^{1+s / d}\right),
$$


where

$$
I_{\nu}^{\mathcal{D}}(t)=\int_{\mathcal{D} \times \mathcal{D}} \frac{1}{|x-y|^{t}} d \nu(x) d \nu(y), \quad 0<t<d .
$$

The measure $\nu$ is a multiple of the Lebesgue measure such that if $\Omega^{*}$ is a fundamental domain for $\Lambda^{*}$ then $\nu\left(\Omega^{*}\right)=1$.

Proof. From Theorem 1 we get that

$$
\begin{aligned}
\mathbb{E}_{\omega \in\left(\mathbb{R}^{d}\right)^{t} N} & \left(E_{s, \Lambda}(\omega)\right)=\frac{2 \pi^{d / 2}|\Lambda|^{-1}}{\Gamma\left(\frac{s}{2}\right)(d-s)} t_{N}^{2} \\
& -\frac{\pi^{s-\frac{d}{2}} \Gamma\left(\frac{d-s}{2}\right)}{\Gamma\left(\frac{s}{2}\right)|\Lambda|} \sum_{\substack{w, w^{\prime} \in \Lambda^{*} \\
w \neq w^{\prime}}} \frac{\kappa_{N}(w) \kappa_{N}\left(w^{\prime}\right)}{\left|w-w^{\prime}\right|^{d-s}}+o\left(t_{N}^{1+\frac{d}{s}}\right) .
\end{aligned}
$$

Observe that

$$
\frac{t_{N}}{N}=\frac{1}{N} \sum_{w \in \Lambda^{*}} \kappa_{N}(w)=\frac{\#\left(\mathbb{Z}^{d} \cap N^{1 / d} A^{t} \mathcal{D}\right)}{N} \longrightarrow\left|A^{t} \mathcal{D}\right|=|\Lambda||\mathcal{D}|, N \rightarrow \infty .
$$

For the other term,

$$
\begin{aligned}
& \sum_{\substack{w, w^{\prime} \in \Lambda^{*} \\
w \neq w^{\prime}}} \frac{\kappa_{N}(w) \kappa_{N}\left(w^{\prime}\right)}{\left|w-w^{\prime}\right|^{d-s}}=\sum_{\substack{w, w^{\prime} \in \Lambda^{*}, w \neq w^{\prime} \\
w, w^{\prime} \in N^{1 / d} \mathcal{D}}} \frac{1}{\left|w-w^{\prime}\right|^{d-s}} \\
= & N^{1+s / d} \frac{1}{N^{2}} \sum_{\substack{z \neq z^{\prime} \in \mathbb{Z}^{d} \\
z, z^{\prime} \in N^{1 / d} A^{t} \mathcal{D}}} \frac{1}{\left|A^{-t}\left(N^{-1 / d} z-N^{-1 / d} z^{\prime}\right)\right|^{d-s}},
\end{aligned}
$$

and

$$
\begin{aligned}
& \lim _{N \rightarrow \infty} \frac{1}{N^{2}} \sum_{\substack{z \neq z^{\prime} \in \mathbb{Z}^{d} \\
z, z^{\prime} \in N^{1 / d} A^{t} \mathcal{D}}} \frac{1}{\left|A^{-t}\left(N^{-1 / d} z-N^{-1 / d} z^{\prime}\right)\right|^{d-s}}=\int_{A^{t} \mathcal{D} \times A^{t} \mathcal{D}} \frac{1}{\left|A^{-t}(x-y)\right|^{d-s}} d x d y \\
& \quad=|\operatorname{det} A|^{2} \int_{\mathcal{D} \times \mathcal{D}} \frac{1}{|x-y|^{d-s}} d x d y=\int_{\mathcal{D} \times \mathcal{D}} \frac{1}{|x-y|^{d-s}} d \nu(x) d \nu(y)=I_{\nu}^{\mathcal{D}}(d-s) .
\end{aligned}
$$

A natural question is now, given a fixed lattice $\Lambda$, to find the optimal $\mathcal{D} \subset \mathbb{R}^{d}$ in the definition of $\kappa^{\mathcal{D}, \Lambda}$. By the result above, all we have to check is what is the domain giving the larger potential $I_{\nu}^{\mathcal{D}}$. The result follows from Riesz rearrangement inequality [10, p. 87].

Theorem 2 (Riesz rearrangement inequality). Given $f, g, H$ nonnegative functions in $\mathbb{R}^{d}$ with $h(x)=H(|x|)$ symmetrically decreasing. Then

$$
\int_{\mathbb{R}^{d}} \int_{\mathbb{R}^{d}} f(x) g(y) H(|x-y|) d x d y \leq \int_{\mathbb{R}^{d}} \int_{\mathbb{R}^{d}} f^{*}(x) g^{*}(y) H(|x-y|) d x d y,
$$


where $f^{*}, g^{*}$ are the symmetric decreasing rearrangements of $f$ and $g$.

We apply the result for $f=g=\chi_{\mathcal{D}}$ getting $f^{*}=g^{*}=\chi_{\mathcal{D}^{*}}$ where $\mathcal{D}^{*}$ is the open ball centered at the origin with $\left|\mathcal{D}^{*}\right|=|\mathcal{D}|$. If $\omega_{d-1}=d \frac{\pi^{d / 2}}{\Gamma\left(\frac{d}{2}+1\right)}$ is the surface measure of the unit ball $\mathbb{S}^{d-1}$ in $\mathbb{R}^{d}$ then

$$
\mathcal{D}^{*}=\left\{x \in \mathbb{R}^{d}: \omega_{d-1}|x|^{d}<d|\mathcal{D}|\right\}=B\left(0, r_{d}\right), \text { with } r_{d}=\left(\frac{d|\mathcal{D}|}{\omega_{d-1}}\right)^{1 / d} .
$$

2.1. An upper bound for the minimal periodic Riesz $s$-energy. It is clear that

$$
\mathcal{E}_{s, \Lambda}(N) \leq \mathbb{E}_{\omega \in\left(\mathbb{R}^{d}\right)^{N}}\left(E_{s, \Lambda}(\omega)\right)
$$

and therefore from the results above we get that $C_{s, d}$ in $(2)$ satisfies, for $0<s<d$,

$$
C_{s, d} \leq-\frac{\pi^{s-\frac{d}{2}} \Gamma\left(\frac{d-s}{2}\right)}{\Gamma\left(\frac{s}{2}\right)|\Lambda|^{1-\frac{s}{d}}} I_{\nu}^{\mathcal{D}}(d-s)
$$

where $\mathcal{D}$ is any bounded domain such that $|\mathcal{D}||\Lambda|=1$. By the discussion above the best choice is to take the set $\mathcal{D}$ to be a ball.

Proposition 3. Let $\Lambda \subset \mathbb{R}^{d}$ be a lattice and

$$
\mathcal{D}=B\left(0, r_{d}\right), \text { with } r_{d}=\left(\frac{d|\Lambda|^{-1}}{\omega_{d-1}}\right)^{1 / d} .
$$

Then $|\mathcal{D}||\Lambda|=1$ and

$$
\frac{\pi^{s-\frac{d}{2}} \Gamma\left(\frac{d-s}{2}\right)}{\Gamma\left(\frac{s}{2}\right)|\Lambda|^{1-\frac{s}{d}}} I_{\nu}^{\mathcal{D}}(d-s)=d\left[2 \pi\left(\frac{d}{\omega_{d-1}}\right)^{1 / d}\right]^{s} \int_{0}^{\infty} \frac{J_{d / 2}^{2}(t)}{t^{1+s}} d t
$$

where according to [9, p.47, (4)]

$$
\begin{gathered}
\int_{0}^{\infty} \frac{J_{d / 2}^{2}(t)}{t^{1+s}} d t=\frac{\Gamma\left(\frac{d-s}{2}\right)}{2^{d+1} \Gamma\left(\frac{d}{2}+1\right) \Gamma\left(\frac{s}{2}+1\right)}{ }_{2} F_{1}\left(\frac{d-s}{2}, \frac{d+1}{2} ; d+1 ; 1\right) \\
=\frac{\Gamma\left(\frac{d-s}{2}\right) \Gamma(d+1) \Gamma\left(\frac{s+1}{2}\right)}{2^{d+1} \Gamma\left(\frac{d}{2}+1\right) \Gamma\left(\frac{s}{2}+1\right) \Gamma\left(\frac{d+s}{2}+1\right) \Gamma\left(\frac{d+1}{2}\right)} .
\end{gathered}
$$

Proof. The proof is an easy computation. We take the normalization of the Fourier transform

$$
\hat{f}(\xi)=\int_{\mathbb{R}^{d}} f(x) e^{-2 \pi i\langle x, \xi\rangle} d x
$$

and then, in distributional sense, if $f_{s}(x)=|x|^{-s}$,

$$
\hat{f}_{s}(\xi)=\frac{\pi^{s-\frac{d}{2}} \Gamma\left(\frac{d-s}{2}\right)}{\Gamma\left(\frac{s}{2}\right)} \frac{1}{|\xi|^{d-s}} .
$$


For any $K \subset \mathbb{R}^{d}$ we have

$$
\begin{gathered}
\int_{K \times K} \frac{1}{|x-y|^{s}} d x d y=\int_{K}\left(f_{s} * \chi_{K}\right)(y) d y=\left(f_{s} * \chi_{K}, \chi_{K}\right) \\
=\left(\hat{f}_{s} \widehat{\chi_{K}}, \widehat{\chi_{K}}\right)=\frac{\pi^{s-\frac{d}{2}} \Gamma\left(\frac{d-s}{2}\right)}{\Gamma\left(\frac{s}{2}\right)} \int_{\mathbb{R}^{d}} \frac{\left|\widehat{\chi_{K}}(\xi)\right|^{2}}{|\xi|^{d-s}} d \xi
\end{gathered}
$$

and the result follows from

$$
\widehat{\chi_{B(0, r)}}(\xi)=r^{d / 2} \frac{J_{d / 2}(2 \pi r|\xi|)}{|\xi|^{d / 2}}
$$

Remark 2. The function

$$
A_{s, d}=-d\left[2 \pi\left(\frac{d}{\omega_{d-1}}\right)^{1 / d}\right]^{s} \frac{\Gamma\left(\frac{d-s}{2}\right) \Gamma(d+1) \Gamma\left(\frac{s+1}{2}\right)}{2^{d+1} \Gamma\left(\frac{d}{2}+1\right) \Gamma\left(\frac{s}{2}+1\right) \Gamma\left(\frac{d+s}{2}+1\right) \Gamma\left(\frac{d+1}{2}\right)},
$$

is such that $A_{0, s}=-1$ and the residue in $d$ is

$$
\lim _{s \rightarrow d}(s-d) A_{s, d}=\omega_{d-1},
$$

as the Epstein zeta functions, and is negative for $0<s<d$. Recall that it has been conjectured (see [4]) that $C_{s, d}$ is given by $\zeta_{\Lambda_{d}}(s)$ for $d=2,4,8,24$, where $\Lambda_{d}$ denotes (respectively) the hexagonal lattice, the $D_{4}$ lattice, the $E_{8}$ lattice and the Leech lattice (scaled to have $\left|\Lambda_{d}\right|=1$ ). The value of $A_{s, d}$ is bigger (i.e. worst) than these conjectured values for $C_{s, d}$ with $d=2,4,8,24$, see figure 1. However, it is observed in [12] that no negative bound was known for $C_{s, d}$ for large dimensions. Indeed, it was proved in [13] (see also [14, 4.4.4.]) that for $0<\delta<1$ and $d$ sufficiently large then

$$
\zeta_{\Lambda}(\delta d)>0
$$

for every lattice $\Lambda$, such that the shortest non-zero vector in $\Lambda$ satisfies

$$
m(\Lambda) \leq \delta \sqrt{\frac{d}{\pi e}}
$$

and this last condition is satisfied by all explicitly known lattices in large dimensions.

\section{REFERENCES}

[1] K. Alishashi, M. S. Zamani. The spherical ensemble and uniform distribution of points on the sphere. Electron. J. Probab. 20 (2015), no. 23, 27 pp.

[2] G. W. Anderson, A. Guionnet, O. Zeitouni, An introduction to random matrices. Cambridge Studies in Advanced Mathematics, 118. Cambridge University Press, Cambridge, 2010.

[3] C. Beltrán, J. Marzo, J. Ortega-Cerdà, Energy and discrepancy of rotationally invariant determinantal point processes in high dimensional spheres, J. of Complexity, 37, 76-109, 2016. 

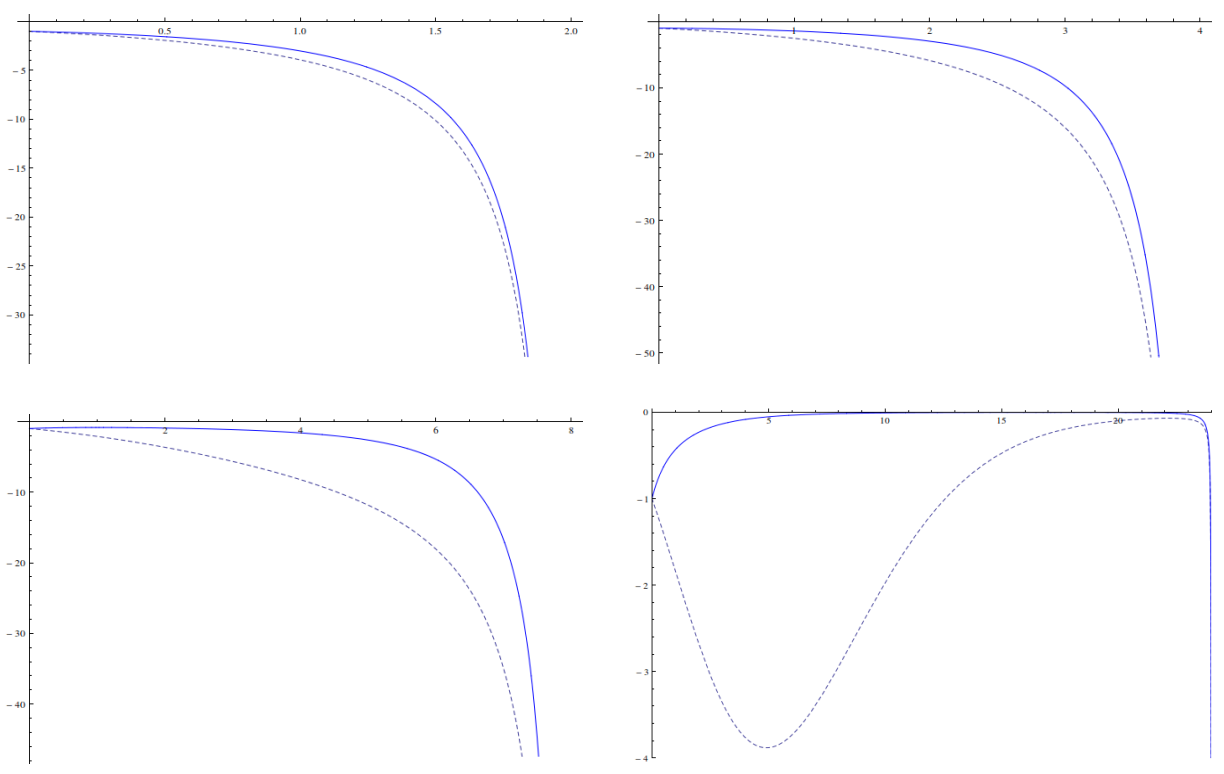

Figure 1. $A_{s, d}$ dashed and $\zeta_{\Lambda_{d}}(s)$ solid for $d=2,4,8,24$

[4] J. S. Brauchart, D. P. Hardin, E. B. Saff. The next-order term for optimal Riesz and logarithmic energy asymptotics on the sphere. Recent advances in orthogonal polynomials, special functions, and their applications, 31-61, Contemp. Math., 578, Amer. Math. Soc., Providence, RI, 2012.

[5] H. Cohn, A. Kumar, Universally optimal distribution of points on spheres, J Am Math Soc 20(1), 99-148, 2007.

[6] D. P. Hardin, E. B. Saff, B. Z. Simanek, Periodic discrete energy for long-range potentials, J. Math. Phys. 55 (2014), no. 12.

[7] D. P. Hardin, E. B. Saff, B. Z. Simanek, Y. Su, Next order energy asymptotics for Riesz potentials on flat tori, arXiv:1511.01552 [math-ph], 2015.

[8] J. Ben Hough, M. Krishnapur, Y. Peres, V. Virág. Zeros of Gaussian Analytic Functions and Determinantal Point Processes. American Mathematical Society, Providence, RI, 2009.

[9] A. Erdélyi, W. Magnus, F. Oberhettinger, F.G. Tricomi. Tables of integral transforms. Vol. II. McGraw-Hill Book Company, Inc., New York-Toronto-London, 1954.

[10] E. H. Lieb, M. Loss, Analysis, Second edition, AMS, Graduate Studies in Mathematics, vol. 14, 2001.

[11] H. L. Montgomery, Minimal theta functions, Glasgow Mathematical Journal, 30(01):75-85, 1988.

[12] P. Sarnak, A. Strömbergsson, Minima of Epstein's Zeta Function and Heights of Flat Tori, Invent. Math., 165 (2006), 115-151.

[13] Terras, A. The minima of quadratic forms and the behavior of Epstein and Dedekind zeta functions, J. Number Theory 12, 258-272 (1980)

[14] Terras, A. Harmonic Analysis on Symmetric Spaces and Applications, Vol. II. Springer-Verlag, Berlin, 1988.

Departament de Matemàtiques i Informàtica, Universitat de Barcelona \& BarCelona Graduate School of Mathematics, Gran Via 585, 08007, Barcelona, Spain

E-mail address: jmarzo@ub.edu 
Departament de Matemàtiques i Informàtica, Universitat de Barcelona \& BarCelona Graduate School of Mathematics, Gran Via 585, 08007, Barcelona, Spain

E-mail address: jortega@ub.edu 